\title{
ENSEÑANZA-APRENDIZAJE CON PROSPECTIVA DE PAZ EN LA FORMACIÓN POLICIAL
}

\author{
TEACHING-LEARNING WITH PROSPECTIVE \\ PEACE IN POLICE TRAINING
}

HÉCTOR BETANCUR GIRALDO*

\begin{abstract}
Recibido: 20 de agosto de 2017 - Aceptado: 20 de septiembre de 2017 - Publicado 31 julio de 2018
\end{abstract} DOI: $10.24142 /$ raju.v13n26a6

\section{Resumen}

La investigación, al ser un ejercicio de naturaleza humana, permite realizar la descripción de una porción de realidad desde su objeto de estudio; por tanto, este trabajo investigativo permitió abordar algunos aportes de la teoría crítica de Frankfurt a través del ejercicio filosófico-reflexivo, orientado a pensar lo social desde lo humano con el fin de transformar una postura tradicional, desde lo crítico, contrastando la heteronimia que seculariza las voluntades humanas, pensando las problemáticas sociales desde el reconocimiento del otro como sujeto y generando acciones encaminadas a la emancipación de los fenómenos tradicionales como es la "Cultura de Guerra" desde la educación policial.

Palabras clave: aprendizaje, enseñanza, policía, paz, proceso.

* Licenciado en Filosofía Analítica, Especialista en Educación, Cultura y Ciencia Política Universidad Nacional Abierta y a Distancia (UNAD), Magíster en Educación Universidad de Medellín. Jefe de Investigación Escuela de Policía Carlos E. Restrepo (ESCER), Estudiante de Sociología Universidad Nacional Abierta y a Distancia (UNAD). Correo electrónico: tobybetan5@ gmail.com 


\section{Abstract}

Research, being an exercise of human nature, allows to realize the description of a portion of reality from its object of study; Therefore, this research work allowed to address some contributions of the critical theory of Frankfurt through the philosophical - reflexive exercise, oriented to think the social from the human in order to transform a traditional posture, from the critical, contrasting heteronomy that secularizes human will, thinking about social issues from the recognition of the other as a subject and generating actions aimed at the emancipation of traditional phenomena such as the "War Culture", from police education.

Keywords: learning, teaching, police, peace, process. 


\section{INTRODUCCIÓN}

La primera condición para la paz es la voluntad de lograrla Juan Luis Vives (1492-1540)

Este trabajo investigativo planteó uno de los desafíos de mayor importancia para el país y para la Policía Nacional de Colombia, el de la paz; por tal motivo, se logró explorar y describir, a partir de las percepciones y experiencias que en la materia se tenía en la institución, la comprensión, el análisis y la formulación de estrategias pedagógicas que, desde la formación policial, se pueden realizar en los procesos de educación policial y que contribuyen al mantenimiento de la paz en Colombia.

Cabe señalar que esta investigación permitió tener un acercamiento a la realidad institucional en materia de conocimientos sobre cultura, cátedra y pedagogía para la paz, y sobre la forma como se desarrollan estos procesos desde la enseñanza-aprendizaje en la formación policial, lo cual permitió generar las condiciones necesarias para la formulación de estrategias que, desde la academia, ayuden a desarrollar acciones sugerentes para la implementación de dicha cátedra para la paz desde la educación en la Policía Nacional de Colombia, asumiendo, de forma estratégica, los retos que enfrentará el Estado y la sociedad de cara al posacuerdo.

Si bien es cierto que la institución es garante del respeto por los derechos humanos y la convivencia pacífica en Colombia, es necesario que los procesos estructurales, como el de formación, deban estar dirigidos a lograr que estos objetivos se cumplan en el país; por tal motivo, es fundamental que los integrantes de la institución posean los conocimientos necesarios en torno a la cultura y pedagogía para la paz, lo cual posibilite llevar a cabo el ejercicio constitucional de cumplir los mandatos que allí están contemplados y poder contribuir a la consolidación de la paz estable y duradera para los colombianos.

Consecuente con este planteamiento, se logró la exploración de las percepciones y conocimientos en materia de cultura, cátedra y pedagogía para la paz que posee el personal policial, sugiriendo estrategias que, desde la formación policial en los procesos de enseñanza-aprendizaje, permitan que el personal posea competencias desde lo social y lo humano, para contribuir al mantenimiento de la cultura pacífica en Colombia.

De acuerdo con los resultados, se logró la creación de políticas institucionales desde la educación policial, con el fin de consolidar acciones 
que acepten el compromiso con la paz en el país a través de la formación de profesionales integrales, conscientes de la importancia de mantener la convivencia pacífica desde su misionalidad, logrando asumir los retos generados por el posacuerdo.

\section{PLANTEAMIENTO DEL PROBLEMA}

Los educadores, más que cualquier otra clase de profesionales, son los guardianes de la civilización

Bertrand Russell (1872-1970)

Abordar los temas relacionados con el posacuerdo en Colombia, desde una prospectiva de paz en la formación policial, permite orientar acciones desde los procesos de enseñanza-aprendizaje fundamentados a partir de una visión humana y social, enfrentando el reto institucional de contribuir a la construcción de la paz y el mantenimiento de las condiciones; por consiguiente, es necesario fortalecer las propuestas que se generan desde los procesos de formación policial y desde su misionalidad constitucional y que permitan ser garantes de la convivencia pacífica en Colombia.

Conviene advertir que las condiciones en que ha discurrido la realidad política y social del país exigen acciones coherentes por parte de las instituciones del Estado, encaminadas al beneficio de la sociedad; más aún cuando, de forma significativa, se han presentado acciones violentas en medio del conflicto armado colombiano, teniendo en medio a la población civil; razón que exige medidas por parte de las instituciones del gobierno, siendo una de ellas la Policía Nacional de Colombia que, desde su misionalidad, debe llevar a cabo acciones en beneficio del mantenimiento de la paz en el posacuerdo mediante mecanismos que permitan su mantenimiento en el país; por tal motivo, este trabajo investigativo puso en escena un tema de relevancia institucional, logrando, desde la formación policial, realizar esfuerzos para contribuir con dicho objetivo.

En ese contexto se debe tener en cuenta la ley 1732 "por la cual se establece la cátedra de la paz en todas las instituciones del país" (República de Colombia, 2014), donde se plantea la necesidad de su aplicabilidad en las Instituciones de Educación Superior "Parágrafo 1. En observancia del principio de autonomía universitaria, cada institución de educación superior desarrollará la Cátedra de la Paz, en concordancia con sus programas académicos y su modelo educativo" (p. 1). 
Si bien es cierto, la formación policial está regida por la Constitución Política de Colombia (1991) y por los parámetros establecidos en la institución mediante la ejecución del Proyecto Educativo Institucional (PEI) de la Policía Nacional de Colombia, cuyos fundamentos van orientados desde un nuevo entorno global; esto permite la flexibilidad en materia de evolución de los modelos educativos y en la fundamentación de los procesos "Enseñanza-Aprendizaje", que se llevan a cabo en la institución; por tanto, en la ley 1732 se faculta a la institución, a través de la autonomía universitaria, para adelantar procesos que permitan la formación en cultura de paz.

Es necesario resaltar que la educación policial universitaria, planteada desde un enfoque que haga frente a las necesidades de la sociedad, debe procurar por responder a las expectativas de convivencia pacífica en el país, con el fin de formar a sus integrantes a partir de referentes teóricos y académicos orientados a la formación de un profesional integral, siendo una educación basada en principios éticos, fundamentada en el respeto por los derechos humanos y la libertad de las personas, logrando llevar a cabo un proceso educativo coherente con las necesidades de la sociedad, la cual requiere que los miembros que la integran, mujeres y hombres, tengan las condiciones óptimas y que reciban la educación necesaria para ejercer dicha labor en el territorio nacional (Policía Nacional de Colombia, 2010).

En este contexto, la Policía Nacional, a través de su institución universitaria, lidera la política educativa y plantea un enfoque que responde a las necesidades de la institución y del país, con el fin de aportar mejores hombres y mujeres a la función de policía, mediante procesos de formación integral del talento humano (p. 42).

Por consiguiente, es fundamental para la educación policial la protección de los derechos humanos a través de acciones que propendan por la convivencia pacífica en el país, siendo un objetivo permanente en los procesos de formación policial, procurando mantener las condiciones necesarias para que dicho objetivo se cumpla en Colombia.

\section{Metodología}

Se realizó una investigación educativa, orientada a la pedagogía para la paz, con un enfoque no solamente basado en la educación si no en lo social desde la formación policial, siendo llevada a cabo en la Escuela de 
Policía Carlos Eugenio Restrepo, unidad adscrita a la Policía Nacional de Colombia (PNC), con la finalidad de analizar la formación policial desde los procesos de enseñanza-aprendizaje de los estudiantes a Técnicos Profesionales en Servicio de Policía, ${ }^{1}$ siendo una investigación de campo, exploratoria, descriptiva, orientada a la formación policial hacia la prospectiva de paz por medio de un proceso realizado con 380 estudiantes, y una selección del $20 \%$ como muestra para dicho estudio.

Se empleó un muestreo aleatorio dividiendo la población en estratos (secciones). Después, en cada estrato se llevó a cabo un muestreo aleatorio simple, con el fin de que todas las secciones (10) quedaran representadas en la aplicación de dicho instrumento. Para el cumplimiento de los objetivos y obtención de los datos se diseñó un instrumento basado en la "Cátedra para la Paz", teniendo en cuenta los criterios del Ministerio de Educación de Colombia, indagando sobre las características y experiencias que en materia de posacuerdo y paz poseían los estudiantes de la escuela para el año 2016.

Antes de aplicar el instrumento se realizó una prueba piloto con diez estudiantes; esta prueba permitió analizar algunos aspectos y características de su diseño, detectándose algunas inconsistencias de formulación, siendo corregidas antes de aplicarlas y siendo avalados por la Dirección Nacional de Escuelas de la Policía Nacional. A su vez, se empleó un cuestionario autoadministrado donde los encuestados respondieron a las preguntas de manera anónima, diligenciaron los formatos de consentimiento informado a los participantes en el estudio, en los que asumieron su voluntad de participar no sin antes revisar que se les garantizara que la información fuera confidencial y fuera utilizada para fines investigativos.

\section{HACIA UNA PEDAGOGÍA PARA LA PAZ EN LA FORMACIÓN POLICIAL}

La pedagogía para la paz en Colombia es una temática que ha emergido en el campo de la Educación, las Ciencias Sociales y Humanas, abordada desde diferentes perspectivas de análisis, ya que su proceso de configuración posee una dinámica compleja, toda vez que los campos de estudio que la han abordado están en estrecha relación con casi la totalidad de las relaciones sociales y humanas que se establecen entre los individuos. Por tanto, algu-

1 Título obtenido por los patrulleros de la Policía Nacional de Colombia. 
nas investigaciones e informes datan sobre la realidad de la pedagogía para la paz y sobre la formación de estrategias en los procesos de posacuerdo en algunos países del mundo que han tenido dicha experiencia, comprendiendo que se trata de una perspectiva poco abordada en las instituciones policiales.

Por tal motivo, Betancur (2015) realiza una investigación sobre el conflicto en la Policía Nacional de Colombia, donde:

Un conflicto armado como el colombiano, que es asumido por ley por parte del Estado colombiano desde el primero de enero del año 1985 y que en su extensa duración por los vastos territorios del país ha sido signado por múltiples matices y transformaciones que han dejado a sus habitantes sumidos en una gran crisis humanitaria (p. 12).

El investigador hace un planteamiento sobre la complejidad del conflicto armado colombiano, teniendo en cuenta que, aunque se han desarrollado algunas investigaciones en diferentes contextos del orden internacional y nacional al respecto, pocas han planteado una salida negociada, con las posibilidades que puede brindar la pedagogía para la paz como forma de solución del conflicto; por tanto, el elemento central de este trabajo fue el de generar soluciones desde la cultura para la paz, con el fin de contrarrestar dicho fenómeno.

Sandoval (2012) realiza un análisis sobre la complejidad de los conflictos armados y las violencias en Latinoamérica, fundamentalmente en México, y revisa algunos factores concretos que, en materia de paz, se han llevado a cabo, hallando, entre otros, el de la dificultad de lograr procesos de paz en la región, aduciendo razones que van desde la descripción de la violencia estructural sistémica, la violencia directa, la violencia cultural y la violencia simbólica, todas ellas presentes en el desarrollo histórico del conflicto armado colombiano.

La historia de la humanidad está conformada por una serie de acontecimientos que han marcado la vida económica, política, social, cultural y educativa de los pueblos del mundo con signos de convivencias pacíficas y también de violencias. Las dinámicas bélicas han dejado millones de pérdidas humanas, destrucción material y un profundo dolor en la humanidad (Sandoval, 2012, p. 18).

De igual modo, este estudio permite comprender que las violencias en la región están signadas por multiplicidad de factores, que van desde lo 
político hasta lo cultural de los pueblos, muchos de ellos inmersos en formas de violencias, otros cuantos en convivencia pacífica; los que tienen formas de violencias han recibido consecuencias funestas y trágicas en torno a lo humano y lo social.

Por tal motivo, se comprendió, a través de este trabajo, la importancia de construir la paz en Colombia por medio del análisis de los ejemplos de la región, y asimilando que:

\begin{abstract}
Se plantea una paz integral porque no tiene que ser una paz solamente reducida a ciertos aspectos y ámbitos de la vida, sino que tiene que ser completa, de un todo y en todos los aspectos, pues de lo contrario hablaremos y tendremos una paz frágil, vulnerable, negativa. Esta paz integral está condicionada a que haya justicia, libertad, respeto a los derechos humanos, a los derechos colectivos de los pueblos indígenas, a la autonomía, interculturalidad, y democracias reales (Sandoval, 2012, p. 30).
\end{abstract}

En concreto, una paz integral requiere del consenso entre el Estado, sus instituciones y la sociedad, con propuestas y estrategias orientadas a disminuir las brechas de injusticia e inequidad social, en donde la academia, a través de la investigación y la educación, genere procesos estructurados que permitan mitigar sus efectos, asumiendo que la institución policial no debe ser ajena a asumir su rol dentro del Estado y aportando a la construcción de la convivencia pacífica en Colombia.

Salamanca (2009) realiza un análisis sobre la violencia tanto directa como estructural; ambas han tenido efecto sobre las dinámicas sociales y sus manifestaciones, razón que ha limitado la posibilidad de diálogo como mecanismo efectivo para solucionar las diferencias entre los individuos en sociedad, sumado a factores tan complejos como el modelo económico y los intereses del poder, el cual mantiene una brecha de desigualdad en la sociedad sobre la cual ejerce su dominio.

La educación, frente a esta problemática, se ha venido planteando el interrogante y reto de cómo hacer para que estas tendencias puedan ser modificadas; es así como nace la Educación para la Paz. Esta se presenta como alternativa innovadora de aprendizaje en capacidad de ajustarse a los cambios coyunturales y dar soluciones pedagógicas a los nuevos retos mundiales (p. 1). 
De acuerdo con esto, la formación policial, desde la prospectiva de paz, debe estar orientada a generar las condiciones necesarias que faciliten la evolución y el tránsito hacia la paz; por tal motivo, la inclusión de la reflexión permite gestar alternativas desde lo humano, social y educativo, con el fin de enfrentarse al reto de mantener la paz en Colombia mediante el ejercicio constitucional encomendado, estando preparada estratégicamente para enfrentar dicha condición; por tanto:

La educación puede llegar a despertar en las personas un sentido crítico que les permite analizar el entorno, la ideología, la religión y la cultura donde se están desenvolviendo. Este proceso de análisis personal posibilita la inserción del hombre en un espacio propio, haciéndole partícipe de su desarrollo como sujeto, actuar de forma consecuente con su criterio y realizar propuestas de cambio si lo cree pertinente (Salamanca, 2009, p. 7).

Finalmente, el establecimiento de estrategias desde la enseñanza-aprendizaje, en materia de cultura y pedagogía para la paz, generan el compromiso permanente en los procesos educativos (incluyendo a quienes en ella intervienen) a través de mecanismos que, desde una postura crítica, faciliten la generación de acciones en beneficio de la sociedad, siendo conscientes del compromiso con la construcción de la convivencia pacífica y articulando lo que plantea la teoría crítica de la sociedad de la Escuela de Frankfurt, al momento de reconocer al otro como sujeto para la comprensión y el diálogo permanente.

\section{DE LA VIOLENCIA A LA CULTURA DE PAZ}

Gutiérrez (2004) aborda las relaciones de poder y su externa influencia desde las formas en que se desarrollan, logrando articular, desde la perspectiva de Bourdieu, la injerencia que puede tener en los sujetos y en las estructuras sociales.

Cuando Bourdieu hace referencias al "objetivismo" y al "subjetivismo", y a la necesidad de tomar lo mejor de ambas perspectivas, sus señalamientos son percibidos e interpretados, la mayoría de las veces, solo como fundamentos de dos momentos analíticos: un primer momento objetivista, en el cual el investigador reconstruye la estructura de relaciones donde se insertan las prácticas, relaciones que son in- 
dependientes de la conciencia y de la voluntad de los agentes, y un segundo momento, donde se intentan captar representaciones, percepciones y vivencias de los protagonistas de esas prácticas (p. 290).

La idea que se plantea define el fenómeno de la violencia de forma objetiva, y permite llegar al interior de las relaciones que se entretejen entre los sujetos en sociedad donde el investigador social las perciba desde la experiencia de vida de quienes las experimentan, llegando allá, al lugar donde convergen los hechos sociales; por tanto, se logran evidenciar aspectos relacionados con el fenómeno de la violencia y se pueden comprender las bases para la construcción y desarrollo de la pedagogía para la paz en la institución, articulando conceptos como el hábitus, donde el sujeto construye sus relaciones de poder, determinadas por la sociedad en la cual está inmerso; entre ellas, la posibilidad de generar una cultura pacifista, razón lógica para comprender la imposición de la violencia o de la paz dentro de la estructura social y de las relaciones de los sujetos que en ella convergen; observando que existe una relación permanente entre el individuo y lo que lo rodea:

¿Cómo es posible escapar a su acción? La acción de violencia simbólica es tanto más fuerte cuanto mayor es el desconocimiento de su arbitrariedad, y uno puede destruir ese poder de imposición simbólica a partir de una toma de conciencia de lo arbitrario, lo que supone el develamiento de la verdad objetiva y el aniquilamiento de la creencia que la sustenta (Gutiérrez, 2004, p. 298).

Por otra parte, Blair (2009) se acerca al concepto de violencia, donde el investigador, a través del análisis del concepto, determina algunas dificultades en materia de conceptualización de la violencia, para lo cual realiza una retrospección del concepto y lo define en un relación tan ligada a la condición humana como la misma historia del mundo; a su vez, resalta que algunas construcciones del concepto han surgido a partir de diferentes apreciaciones, siendo las de los medios de comunicación las más asimiladas por la sociedad.

Por esta vía hemos conocido más sobre el país de lo que había aportado el estudio de otras problemáticas dándole, sin duda, la razón a Gonzalo Sánchez cuando plantea que: guerra y política, orden y violencia, violencia y democracia y, en el límite, vida y muerte, son algunas de las múltiples oposiciones y complementariedades a partir 
de las cuales se hace descifrable la historia colombiana (Sánchez citado en Blair, 2009, p. 32).

Cabe señalar que son varios los fenómenos sociales y las problemáticas en las que se han presentado distintas formas de violencia en Colombia, diferentes matices y estructuras que han complejizado el panorama y desde donde se ha construido la historia colombiana; sin embargo, los estudios sobre la violencia deben tener, como punto de partida, la salida de la violencia a la paz a través de la reflexión sobre los efectos devastadores de la violencia en sociedad, con el fin de transformarlos por medio de posturas pacifistas desde la pedagogía, la educación y la cultura para la paz en Colombia.

\section{APORTES DE LA TEORÍA CRÍTICA PARA FORMAR CULTURA PARA LA PAZ}

Teniendo en cuenta los aportes de la teoría crítica de la sociedad, esta permite realizar un acercamiento al fenómeno desde diferentes posiciones, las cuales pueden ser asumidas por la institución para generar la cultura para la paz.

Seoane (2010) plantea:

El actor orientado por intereses emancipatorios puede actuar en cualquier situación social. Empero, la teoría considera que hay lugares privilegiados para la práctica transformadora, lugares estratégicos por el amplio alcance socializante. Entre los mismos cabe mencionar a los medios de comunicación (información) y las instituciones educativas, puesto que actualmente constituyen importantes centros de subjetivación (p. 28).

Por tanto, se puede comprender que la formación de sujetos con pensamiento crítico puede generar acciones encaminadas al fortalecimiento de la conciencia en materia de la importancia de la construcción de la paz, logrando transformaciones del ideario social que permitan la emancipación de la cultura guerrerista y rechazando, entre otras, las posturas de los medios de comunicación encargados de distorsionar la realidad. Con esto se propone llevar a cabo acciones pedagógicas para la reconciliación y la convivencia 
pacífica, mediante propuestas educativas que permitan intervenir en los contextos sociales más golpeados por la violencia.

De igual forma, los retos que se plantee la institución deben estar ligados a la academia y la investigación, optando por generar soluciones como las que propone el pensamiento crítico de Frankfurt, cuando determina:

En estos lugares la teoría se propone una democratización a fondo, conforme a una distribución lo más equitativa posible de los diferentes capitales mencionados en este trabajo. En otros términos, la actitud crítica y dialógica busca incansablemente distribuir el poder entre todos los miembros de una sociedad, procurando, en todo momento, quebrar las relaciones de dominación (Seoane, 2010, p. 28).

Sin duda alguna, pensar en una democratización demanda que sus instituciones realicen acciones basadas en la inclusión y la equidad, logrando convocar un trabajo con todos los sectores de la sociedad civil entre los que se encuentran las poblaciones vulnerables que, para el posconflicto, incluye también a las víctimas. Sin embargo, el pensamiento crítico de la sociedad de la Escuela de Frankfurt define que tanto el individualismo como el sociologismo corresponden a un momento histórico con situaciones muy concretas y que obedecen, en su estructura, a algunas posiciones contradictorias, pero que la esencia, y donde se hace puntual su aplicabilidad en la realidad colombiana, se da cuando empiezan a definirse las raíces históricas de los pensamientos doctrinarios que dan origen a la violencia y la confrontación.

Con esto se deduce, desde la concepción de la antropología filosófica y de los planteamientos sociológicos contemporáneos, la existencia de un pensamiento dialéctico hipostasiado donde simplemente se sitúa al ser humano en la categoría de egoísta o altruista, y se define como condición innata y natural, cuando en términos de Max Horkheimer se refiere a la tendencia de carácter liberal que tiene la particularidad de interpretar al hombre como un sujeto individualista que no posee un sentido social, pero que termina beneficiando con sus acciones al colectivo; comprendiendo que la moderna antropología filosófica delimita el intento de encontrar una norma rectora que le otorgue sentido a la vida de ese individuo en su momento histórico actual, analizando las variables que se presentan en la evolución, tanto del individuo como de su medio y momento histórico; por tanto, se constituye en el punto de reflexión acerca de las condiciones en las que el sujeto debe repensarse para mantener una actitud y voluntad pacifista en sociedad. 
De acuerdo con Friedman (2000):

De esta manera, para los frankfurtianos, el sociologismo expresa un momento de verdad histórica, a saber, la triste verdad de la sociedad unidimensionalizante, o administrada en la versión de Horkheimer y Adorno, de nuestro tiempo. No se trata de una verdad eterna y por consiguiente inmutable, sino de una verdad histórica, de una verdad en la cual el hombre se autoanula en sus propias creaciones, sucumbe a ellas (p. 201).

Del examen anterior se pueden generar criterios de selección de las estrategias educativas orientadas a la construcción de una pedagogía para la paz, que correspondan a la realidad histórica y que apunten a la solución de las necesidades del medio, que visibilicen la posibilidad de aplicación de la teoría crítica en beneficio de la convivencia pacífica para la sociedad colombiana.

\section{CONCLUSIONES}

Se lograron determinar las acciones que, en materia de formación policial, debe realizar la institución en los procesos estructurales como el de enseñanza-aprendizaje, llevando a cabo acciones que den cumplimiento al mandato constitucional sobre la paz como derecho y deber de obligatorio cumplimiento; por tanto, el Estado colombiano y sus instituciones son las encargadas de generar los espacios necesarios para el aprendizaje, la reflexión y el diálogo permanente sobre la convivencia pacífica en Colombia.

El personal policial conoce y comprende la importancia de la ley 1732, aunque se hace indispensable desarrollar estrategias de divulgación para analizar y entender la norma en la institución, resaltando que el personal en proceso de formación policial se interesó por la misma a través del proceso investigativo, comprendiendo la importancia para el país y para la institución de la educación para la paz.

En materia de comprensión de los aspectos relacionados con la cátedra de paz se crea la necesidad de generar contextos de reflexión que contribuyan a la convivencia pacífica, siendo aspectos asimilados por parte del personal del estudio; sin embargo, existe un nivel de desconocimiento del tema en algunos aspectos relacionados con la obligatoriedad de impartir dicha cátedra por parte de la institución. 
Se deben optimizar los mecanismos de divulgación, socialización y cobertura que permitan informar sobre los conocimientos de la cátedra de la paz en la institución a partir de consideraciones para fundamentar y aplicar en la formación policial en los procesos de enseñanza-aprendizaje, para aportar a la construcción de la paz desde la educación policial.

En cuanto a los compromisos con la pedagogía, la cultura, la cátedra para la paz, la responsabilidad y la aplicabilidad se evidenció en su aprobación; sin embargo, existen dudas sobre si es pertinente o no su utilidad en la formación policial, o sobre si la institución debe asumir su incorporación; además, se evidenció que apoyar la paz y ayudar a consolidarla son elementos puntuales sobre los que se hizo énfasis al personal del estudio.

Se planteó el fortalecimiento de la reconciliación, el perdón y la superación del rencor como elementos fundamentales para fortalecer la construcción de la paz; sin embargo, la formación policial requiere que se agregue al currículo dicha cátedra, siendo direccionada hacia la convivencia pacífica, resaltando el compromiso que debe asumir la institución con el mantenimiento de las condiciones necesarias para mantener la paz en Colombia.

\section{RECOMENDACIONES}

Se requiere del compromiso permanente de la Policía Nacional de Colombia a través de la Dirección Nacional de Escuelas, con el diseño y la fundamentación de las estrategias académicas desde la formación policial en los procesos de enseñanza-aprendizaje, que den cumplimiento al mandato constitucional de mantener la paz como derecho de obligatorio cumplimiento en Colombia, facilitando la construcción de escenarios para la convivencia pacífica y su continuidad en el país.

Es fundamental que la Policía Nacional de Colombia establezca políticas educativas de estudio y análisis permanente de la ley 1732 con el fin de fortalecer la formación para la paz en la institución, divulgando y socializando los aspectos que conforman dicha ley a través de la creación de espacios para la reflexión, el pensamiento crítico y el diseño de acciones que contribuyan con la construcción de la paz en el país.

En materia de normatividad, para la construcción de la paz en Colombia se recomiendan estrategias de estudio permanentes de las normas y leyes en los contextos internacional, nacional, regional y local, analizando los mecanismos educativos de la institución que estimulen la convivencia pacífica en el país, comprometiendo al personal a través de la educación y cultura de 
la paz, brindando la posibilidad de participar en el diseño de estrategias para mantener la convivencia pacífica a través de la aplicabilidad de la misionalidad institucional y de contribuir en el progreso de la sociedad y de sus gentes.

A partir de los resultados de la investigación, la institución generó políticas para la consolidación de la formación policial con prospectiva para la paz, mediante estrategias interdisciplinarias orientadas desde las Ciencias Humanas y Sociales, creando espacios para la reflexión y la convivencia pacífica en el país, comprendiendo aspectos como son el perdón, la reconciliación y la superación del rencor, entre otras manifestaciones inmersas en el ideario social colombiano producto de la confrontación armada, aportando a la convivencia pacífica y a la construcción de la paz en Colombia.

\section{REFERENCIAS}

Betancur, H. (2015). Caracterización de las estudiantes de la Escuela de Policía Carlos Eugenio Restrepo víctimas del conflicto armado colombiano año 2015. Antioquia. Colombia. Recuperado de http://www.repositorio.policia.edu.co/bitstream/001/1012/2/S-2016-007137-ESCER.pdf

Blair, E. (2009). Aproximación teórica al concepto de violencia: avatares de una definición. Política y Cultura, (32), 9-33.

Bourdieu, P., y Passeron, J. C. (1977). La reproducción. Elementos para una teoría del sistema de enseñanza. Barcelona: Laia.

Friedman, G. (2000). La filosofía política de la Escuela de Frankfurt. México: Fondo de Cultura Económica.

Gutiérrez, A. B. (2004). Poder, hábitus y representaciones: recorrido por el concepto de violencia simbólica en Pierre Bourdieu. Revista Complutense de Educación, 15(1), 289-300. Recuperado de http://revistas.ucm.es/ index.php/RCED/article/view/RCED0404120289A

Policía Nacional de Colombia (2010). Capítulo IV: la educación como fundamento de la actuación del hombre y mujer policía. En Lineamiento de Política 1. Direccionamiento policial basado en el humanismo con responsabilidad. Bogotá: Imprenta Nacional de Colombia.

República de Colombia (1991). Constitución Política de Colombia. Recuperado de http://www.procuraduria.gov.co/guiamp/media/file/Macroproceso\%20Disciplinario/Constitucion_Politica_de_Colombia.htm

República de Colombia (2014). Ley 1732. Recuperado de http:// wsp.presidencia.gov.co/Normativa/Leyes/Documents/LEY\%201732\%20 DEL\%2001\%20DE\%20SEPTIEMBRE\%20DE\%202014.pdf 
Salamanca, A. (2009). Educación para la paz. Praxis, 5(1), 17-32. Recuperado de http://revistas.unimagdalena.edu.co/index.php/praxis/article/ view/89

Sandoval, E. A. (2012). Estudios para la paz, la interculturalidad y la democracia. Ra Ximhai, 8(2), 17-37. Recuperado de http://revistas.unam.mx/ index.php/rxm/article/view/32929

Seoane, J. B. (2010). Contribución a una teoría crítica dialógica. Revista Venezolana de Economía y Ciencias Sociales, 16(3), 13-31. Recuperado de www.redalyc.org/pdf/177/17731133002.pdf 